\title{
REVIEW
}

\section{Physical activity and all cause mortality in women: a review of the evidence}

\author{
Y Oguma, H D Sesso, R S Paffenbarger Jr, I-M Lee
}

Br J Sports Med 2002;36:162-172

A computer assisted literature search was performed (Medline, 1966-2000) to examine the association of physical activity with all cause mortality in women. It was concluded that, by adhering to current guidelines for physical activity and expending about $4200 \mathrm{~kJ}$ of energy a week, women can postpone mortality. The magnitude of benefit experienced by women is similar to that seen in men.

See end of article for authors' affiliations

Correspondence to: Dr Oguma, College Alumni Health Study, Department of Epidemiology, Harvard School of Public Health, 677 Huntington Avenue, Boston, MA 02115 , USA yoguma@hsph.harvard.edu

Accepted

11 December 2001
$\mathrm{T}$ here is a large body of epidemiological evidence pointing to the health benefits of regular physical activity. ${ }^{1-3}$ Among these health benefits is increased longevity or the postponement of premature mortality. Before the last decade, much of the evidence has come from studies conducted in men. For example, a review of the association of physical activity with all cause mortality published in 1997 discussed 13 studies, only three of which included women. ${ }^{4}$ Recently, however, several additional studies have examined the relation of physical activity to death rates in women.

A search of the medical literature elicited no systemic reviews of the association of physical activity with all cause mortality specifically in women. We were interested to examine this for several reasons. Although favourable physiological changes resulting from physical activity may be expected to occur in both sexes, the magnitude of benefit for averting premature mortality may be different. Men and women have different patterns of physical activity, with men expending more energy and being more likely to engage in vigorous kinds of activities. Men are also more likely to be physically active in their jobs; women are more likely to be involved in household chores and activities around child rearing. In addition, women go through the menopause, with striking changes in levels of reproductive hormones. Physical activity before and after the menopause may have different influences on subsequent mortality, because the onset of the menopause is associated with a less favourable cardiovascular risk factor profile.

We therefore undertook this review to examine the association of physical activity with all cause mortality in women. In addition to confirming that physical activity is indeed associated with lower death rates in women, we wanted to compare the magnitude of association observed with that seen in studies of men. We also attempted to address practical problems for healthcare providers, such as the amount, inten- sity, and duration of activity required for postponement of mortality.

\section{METHODS}

To identify papers for this review, we conducted a literature search using Medline (US National Library of Medicine) to look for papers published between January 1966 and December 2000. The keywords used were related to physical activity (physical activity, physical fitness, cardiorespiratory fitness, exercise, or walking) and mortality (mortality, death, or fatal outcome).

For inclusion in this review, the papers had to present results specifically for women, use the end point of all cause mortality (rather than cause specific mortality, such as cardiovascular disease mortality), and examine the association of physical activity or fitness with all cause mortality. We excluded review papers and papers not published in the English language because we did not have the facility to translate papers written in other languages. In addition, we checked the reference lists of papers included in this review, as well as review papers not included, to search for other eligible papers not identified by our computerised literature search. By these means, we identified 38 papers.

Two authors independently reviewed each of the papers and summarised the data (table 1). In two circumstances, ${ }^{5-8}$ the same data were used to examine the association of physical fitness and all cause mortality in women. We present these data only once in table 1 , choosing the publication in which the data were analysed in more detail. ${ }^{68}$

\section{RESULTS}

A total of 37 prospective cohort studies and one retrospective cohort study that examined the association between physical activity or fitness and all cause mortality in women were identified (table 1). Most of the studies were conducted in western, industrialised countries, with half (19 studies) being based in the United States. The Aerobics Center Longitudinal Study ${ }^{5-8}$ and the Framingham Heart Study $y^{9-12}$ each provided the data for four publications, comprising $21 \%$ of the studies reviewed. Sixteen (42\%) studies were based in Europe, including five from Finland ${ }^{13-17}$ and four from Germany. ${ }^{18-21}$ The remaining three studies were based in Canada ${ }^{22}{ }^{23}$ and Israel. ${ }^{24}$

Most $(32(86 \%))$ of the studies focused exclusively on physical activity, while four assessed only physical fitness ${ }^{61625}$ and another two investigated both physical activity and physical fitness. ${ }^{142}$ Of the 34 studies investigating physical activity, 13 captured total physical activity and 23 measured leisure time physical activity (the sum 
Table 1 Summary of studies examining the association between physical activity or fitness and all cause mortality in women

\begin{tabular}{|c|c|c|c|c|c|c|c|c|}
\hline $\begin{array}{l}\text { Author and year, country } \\
\text { study design }\end{array}$ & $\begin{array}{l}\text { No of } \\
\text { women }\end{array}$ & $\begin{array}{l}\text { Age } \\
\text { (years) }\end{array}$ & $\begin{array}{l}\text { Duration } \\
\text { (years)* }\end{array}$ & $\begin{array}{l}\text { No of } \\
\text { deaths }\end{array}$ & Assessment of physical activity & Main results $\ddagger$ & Factors adjusted for & Other comments \\
\hline $\begin{array}{l}\text { Andersen 2000, Denmark } 26 \\
2 \text { prospective cohort studies } \\
\text { combined } \\
\text { (Copenhagen City Heart } \\
\text { Study; Glostrup Population } \\
\text { Studies) }\end{array}$ & 13375 & $20-93$ & $14(5) \dagger$ & 2881 & $\begin{array}{l}2 \text { questions on LTPA and OPA in the } \\
\text { previous year, with } 4 \text { response options } \\
\text { for each question, assessed by } \\
\text { questionnaire. }\end{array}$ & $\begin{array}{l}v \text { inactive } L T P A \\
\text { Light: } R R=0.65, p<0.001 \\
\text { Moderate: } R R=0.59, p<0.001 \\
\text { Vigorous: } R R=0.64, p=0.20 \\
\text { Among subjects with light, moderate, and heavy LTPA, } \\
\text { Sports participation: } R R=0.47(0.34 \text { to } 0.66) \\
v \text { sitting OPA } \\
\text { More active OPA: } R R=0.86(0.74 \text { to } 0.99)\end{array}$ & $\begin{array}{l}\text { Age, educational level, } \\
\text { blood pressure, lipid } \\
\text { levels, BMI, and smoking }\end{array}$ & $\begin{array}{l}\text { Adjustment for chronic } \\
\text { disease did not change } \\
\text { findings. } \\
\text { Inverse association } \\
\text { observed for women } \\
\text { aged } 20-44,45-64 \text {, } \\
\text { and } \geqslant 65 \text {. }\end{array}$ \\
\hline $\begin{array}{l}\text { Hirvensalo 2000, Finland }{ }^{13} \\
\text { Prospective cohort study } \\
\text { (Evergreen Project at } \\
\text { Jyvaskyla) }\end{array}$ & 718 & $65-84$ & 8 & 225 & $\begin{array}{l}\text { Assessed by interview, using a question } \\
\text { with } 6 \text { response options, plus questions } \\
\text { about participation in a list of sports. } \\
\text { Women were then classified as } \\
\text { sedentary or active. } \\
\text { Mobility was defined as being able to } \\
\text { walk } 2 \mathrm{~km} \text { and climb one flight of stairs } \\
\text { without difficulty. }\end{array}$ & $\begin{array}{l}v \text { mobile-active } \\
\text { Mobile-sedentary: } R R=0.87(0.55 \text { to } 1.40) \\
\text { Impaired-active: } R R=1.72(1.10 \text { to } 2.70) \\
\text { Impaired-sedentary: } R R=2.63(1.82 \text { to } 3.81)\end{array}$ & $\begin{array}{l}\text { Age, marital status, } \\
\text { education, presence of } \\
\text { asthma, diabetes, } \\
\text { neurological diseases, } \\
\text { stroke, mental diseases, } \\
\text { CVD, musculoskeletal } \\
\text { diseases, smoking, and } \\
\text { past physical activity }\end{array}$ & \\
\hline $\begin{array}{l}\text { Stessman } 2000, \text { Israe }^{24} \\
\text { Prospective cohort study } \\
\text { (Jerusalem } 70 \text { year } \\
\text { Longitudinal Study) }\end{array}$ & 207 & 70 & 6 & 24 & $\begin{array}{l}1 \text { question on LTPA with } 4 \text { response } \\
\text { options, assessed by interview. }\end{array}$ & $\begin{array}{l}v<4 \text { hours walking/week } \\
\text { Walks about } 4 \text { hours/week: } R R=0.28 \text { (0.05 to } 1.47) \\
\text { Sports activity } \geqslant 2 \times / \text { week: } R R=0.91(0.26 \text { to } 3.19) \\
\text { Walks } \geqslant 1 \text { hour/day: } R R=0.17(0.02 \text { to } 1.53) \\
p \text { for trend }=0.17\end{array}$ & $\begin{array}{l}\text { Smoking, economic } \\
\text { hardship, and pre-existing } \\
\text { medical conditions }\end{array}$ & \\
\hline $\begin{array}{l}\text { Lee 2000, US } \\
\text { Prospective cohort study } \\
\text { (US Longitudinal Study of } \\
\text { Aging) }\end{array}$ & $\begin{array}{l}7527 \\
\text { (both } \\
\text { sexes) }\end{array}$ & $\geqslant 70$ & 7 & $\begin{array}{l}2870 \\
\text { (both } \\
\text { sexes) }\end{array}$ & $\begin{array}{l}\text { "Compared to other people your age, } \\
\text { would you say you are physically more } \\
\text { active, less active, or about as active?" } \\
\text { "Do you feel that you get as much } \\
\text { exercise as you need, or less than you } \\
\text { need?" }\end{array}$ & $\begin{array}{l}v \text { a lot more active than peers } \\
\text { More active: } R R=0.99 \\
\text { About as active: } R R=1.18, p<0.1 \\
\text { Less active: } R R=1.40, p<0.01 \\
v \text { getting as much exercise as needed } \\
\text { Less than needed: } R R=1.05\end{array}$ & $\begin{array}{l}\text { Sociodemographic } \\
\text { characteristics, } \\
\text { functioning, diseases, use } \\
\text { of services, and self } \\
\text { assessed general and } \\
\text { mental health }\end{array}$ & \\
\hline \multirow[t]{2}{*}{$\begin{array}{l}\text { Haapanen } 2000, \\
\text { Northeastern Finland'4 } \\
\text { Prospective cohort study }\end{array}$} & 1122 & $35-63$ & 16 & 87 & $\begin{array}{l}\text { Energy expenditure index assessed by } \\
\text { questionnaire with } 23 \text { questions on } \\
\text { LTPA, household chores and } \\
\text { commuting. }\end{array}$ & $\begin{array}{l}v>1500 \mathrm{kcal} / \text { week } \\
800.1-1500 \mathrm{kcal} / \text { week: } R R=0.59(0.30 \text { to } 1.18) \\
0-800 \mathrm{kcal} / \text { week: } R R=1.27(0.69 \text { to } 2.34)\end{array}$ & $\begin{array}{l}\text { Age, employment status, } \\
\text { marital status, perceived } \\
\text { health status, smoking, } \\
\text { and alcohol consumption }\end{array}$ & \\
\hline & & & & & $\begin{array}{l}\text { Single-item self assessment of LTPA. } \\
\text { Single-item self assessment of physical } \\
\text { fitness compared with age-mates. } \\
\text { Single-item self assessment of } 2 \mathrm{~km} \\
\text { walking ability. } \\
\text { Single-item self assessment of ability to } \\
\text { climb several flights of stairs. }\end{array}$ & $\begin{array}{l}v \text { vigorous activity } \geqslant 1 / \text { week } \\
\text { No or light intensity: } R R=1.61(0.89 \text { to } 2.92) \\
v \text { better physical fittess } \\
\text { Similar: } R R=0.82(0.41 \text { to } 1.65) \\
\text { Worse: } R R=1.71(0.72 \text { to } 4.05) \\
v \text { no difficulties wallking } 2 \mathrm{~km} \\
\text { At least some difficulties: } R R=1.45 \text { (0.78 to } 2.70) \\
v \text { no difficulties climbing stairs } \\
\text { At least some difficulties: } R R=2.39(1.25 \text { to } 4.60)\end{array}$ & & \\
\hline $\begin{array}{l}\text { Dorn 1999, USA } 41 \\
\text { Prospective cohort study } \\
\text { (Buffalo Blood Pressure } \\
\text { Study) }\end{array}$ & 763 & $15-95$ & 29 & 276 & $\begin{array}{l}\text { LTPA and OPA assessed in interview by } \\
28 \text { questions on time spent sleeping, } \\
\text { sitting, driving, standing, caring or } \\
\text { lifting, walking, gardening, exercising } \\
\text { and playing sports. }\end{array}$ & $\begin{array}{l}\text { For every } 1 \mathrm{kcal} / \mathrm{kg} / \text { hour of physical activity, } \\
\qquad 60 \text { years: } R R=1.05(0.59 \text { to } 1.87) \\
\geqslant 60 \text { years: } R R=1.04(0.62 \text { to } 1.74)\end{array}$ & $\begin{array}{l}\text { Age, education, smoking, } \\
\text { and mean arterial blood } \\
\text { pressure }\end{array}$ & \\
\hline
\end{tabular}

Prospective cohort study
Buffalo Blood Pressure

Study)
6 response options, plus questions

mpaired-active: $R R=1.72(1.10$ to 2.70$)$ without difficulty.

$<4$ hours walking/week

Sociodemographic

"Do you feel that you get as much $r$ geting

$800.1-1500 \mathrm{kcal} /$ week: $R R=0.59(0.30$ to 1.18$)$

LTPA, household chores and $\quad 0-800 \mathrm{kcal} /$ week: RR $=1.27(0.69$ to 2.34$)$

Single-item self assessment of LTPA $\quad v$ vigorous activity $\geqslant 1 /$ week

$=1.61(0.89$ to 2.92$)$

better physical fitness

Single-item self assessment of $2 \mathrm{~km} \quad v$ no difficulties walking $2 \mathrm{~km}$

Single-item self assessment of ability to $v$ no difficulties climbing stairs

LTPA and OPA assessed in interview by For every $1 \mathrm{kcal} / \mathrm{kg} /$ hour of physical activity,

28 questions on time spent sleeping, $\quad<60$ years: $R R=1.05(0.59$ to 1.87$)$

, education, smoking, ean arterial blood $\geqslant 60$ years: $R R=1.04(0.62$ to 1.74$)$ 


\begin{tabular}{|c|c|c|c|c|c|c|c|c|}
\hline $\begin{array}{l}\text { Author and year, country } \\
\text { study design }\end{array}$ & $\begin{array}{l}\text { No of } \\
\text { women }\end{array}$ & $\begin{array}{l}\text { Age } \\
\text { (years) }\end{array}$ & $\begin{array}{l}\text { Duration } \\
\text { (years)* }\end{array}$ & $\begin{array}{l}\text { No of } \\
\text { deaths }\end{array}$ & Assessment of physical activity & Main results $\ddagger$ & Factors adjusted for & Other comments \\
\hline $\begin{array}{l}\text { Sherman 1999, USA9 } \\
\text { Prospective cohort study } \\
\text { (Framingham Heart Study) }\end{array}$ & 1410 & $30-62$ & 16 & 522 & $\begin{array}{l}\text { Time spent sleeping, resting, or } \\
\text { engaging in light, moderate, or heavy } \\
\text { physical activity assessed by interview } \\
11-27 \text { years previously and at } \\
\text { baseline. }\end{array}$ & $\begin{array}{l}11-27 \text { years previously, per MET-hour/day } \\
R R=1.01(0.96 \text { to } 1.07) \\
\text { At baseline, per MET-hour/day } \\
\text { RR }=0.84(0.76 \text { to } 0.93)\end{array}$ & $\begin{array}{l}\text { Age, systolic blood } \\
\text { pressure, total cholesterol, } \\
\text { smoking, relative weight, } \\
\text { glucose intolerance, LVH, } \\
\text { COPD, and cancer }\end{array}$ & \\
\hline \multirow[t]{2}{*}{$\begin{array}{l}\text { Villeneuve 1998, Canada }{ }^{22} \\
\text { Prospective cohort study } \\
\text { (Canada Fitness Survey) }\end{array}$} & 8196 & $20-69$ & 7 & 502 & $\begin{array}{l}\text { Average daily energy expenditure in } \\
\text { the past } 12 \text { months on LTPA estimated } \\
\text { from modified Minnesota LTPA } \\
\text { questionnaire based on a subset of the } \\
19 \text { most frequently reported activities, } \\
\text { in KKD (kcal/kg body weight/day). }\end{array}$ & 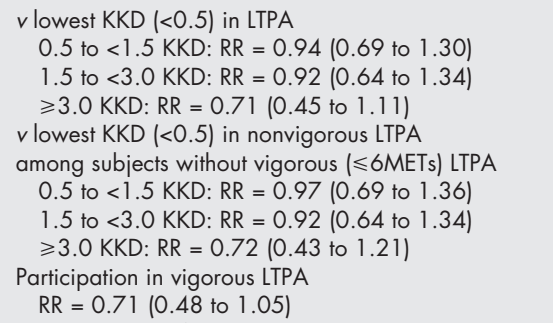 & Age and smoking & \\
\hline & & & & & $\begin{array}{l}3 \text { levels of fitness based on Canadian } \\
\text { Aerobic Fitness Test. }\end{array}$ & $\begin{array}{l}v \text { recommended fitness level (both sexes) } \\
\text { Minimum: } R R=1.02(0.69 \text { to } 1.51) \\
\text { Undesirable: } R R=1.52(0.72 \text { to } 3.18)\end{array}$ & Age, sex, and smoking & $\begin{array}{l}\text { Sex-specific results not } \\
\text { presented for fitness. }\end{array}$ \\
\hline $\begin{array}{l}\text { Weller 1998, Canada } 23 \\
\text { Prospective cohort study } \\
\text { (Canada Fitness Survey) }\end{array}$ & 6620 & $\geqslant 30$ & 7 & 449 & $\begin{array}{l}\text { Average daily energy expenditure on } \\
\text { all activities estimated from modified } \\
\text { Minnesota LTPA questionnaire in KKD } \\
\text { (kcal/kg body weight/day). }\end{array}$ & $\begin{aligned} v \text { lowest } K K D \text { quartile }(Q 1) \text { of leisure activity } \\
Q 2: R R=0.91(0.66 \text { to } 1.25) \\
Q 3: R R=0.94(0.72 \text { to } 1.23) \\
\text { Q4: RR }=0.89(0.67 \text { to } 1.17) \\
v \text { lowest } K K D \text { quartile }(Q 1) \text { of non-leisure activity } \\
Q 2: R R=0.66(0.50 \text { to } 0.87) \\
Q 3: R R=0.68(0.51 \text { to } 0.89) \\
Q 4: R R=0.71(0.50 \text { to } 0.87)\end{aligned}$ & Age & $\begin{array}{l}\text { Adjustment for marital } \\
\text { status, educational level, } \\
\text { income, self reported } \\
\text { health status, and } \\
\text { smoking did not change } \\
\text { findings }\end{array}$ \\
\hline \multirow[t]{5}{*}{$\begin{array}{l}\text { Kujala 1998, Finland } 15 \\
\text { Prospective cohort study } \\
\text { (Finish Twin Study) }\end{array}$} & 7977 & $25-64$ & 18 & 424 & $\begin{array}{l}3 \text { levels of LTPA plus calculated MET } \\
\text { index assessed by questionnaire on } \\
\text { type of activity, frequency, duration, } \\
\text { and intensity. }\end{array}$ & $\begin{array}{l}v \text { sedentary (both sexes) } \\
\text { Occasional exercisers: } R R=0.80(0.69 \text { to } 0.91) \\
\text { Conditioning exercisers: } R R=0.76(0.59 \text { to } 0.98) \\
\text { among same sex twin pairs discordant for death, }\end{array}$ & $\begin{array}{l}\text { Age, sex, smoking, } \\
\text { occupation, and alcohol } \\
\text { use }\end{array}$ & $\begin{array}{l}\text { Women were free of } \\
\text { chronic diseases }\end{array}$ \\
\hline & & & & & & $v$ sedentary (among 148 female twin pairs) & & \\
\hline & & & & & & Occasional exercisers: $R R=0.66$ & $\begin{array}{l}\text { Age, smoking, } \\
\text { occupation, and alcohol }\end{array}$ & \\
\hline & & & & & & Conditioning exercisers: $R R=0.24$ & use. & \\
\hline & & & & & & $\begin{array}{l}\text { v lowest MET quintile (Q1) (among } 434 \text { twin pairs of } \\
\text { both sexes) } \\
\text { Q2: RR }=0.85 \\
\text { Q3: RR }=0.72 \\
\text { Q4: RR }=0.68 \\
\text { Q5: RR }=0.60 \\
\text { p for trend }=0.04\end{array}$ & $\begin{array}{l}\text { Age, sex, smoking, } \\
\text { occupation, and alcohol } \\
\text { use }\end{array}$ & \\
\hline
\end{tabular}




\begin{tabular}{|c|c|c|c|c|c|c|c|c|}
\hline $\begin{array}{l}\text { Author and year, country } \\
\text { study design }\end{array}$ & $\begin{array}{l}\text { No of } \\
\text { women }\end{array}$ & $\begin{array}{l}\text { Age } \\
\text { (years) }\end{array}$ & $\begin{array}{l}\text { Duration } \\
\text { (years) }^{*}\end{array}$ & $\begin{array}{l}\text { No of } \\
\text { deaths }\end{array}$ & Assessment of physical activity & Main results $\ddagger$ & Factors adjusted for & Other comments \\
\hline $\begin{array}{l}\text { Bath 1998, UK }{ }^{31} \\
\text { Prospective cohort study } \\
\text { (Nottingham Longitudinal } \\
\text { Study of Activity and } \\
\text { Ageing) }\end{array}$ & $\begin{array}{l}1042 \\
\text { (both } \\
\text { sexes) }\end{array}$ & $\geqslant 65$ & 12 & $\begin{array}{l}369 \\
\text { (women) }\end{array}$ & $\begin{array}{l}\text { Interview asking about outdoor } \\
\text { productive activities, indoor productive } \\
\text { activities, walking, shopping, leisure } \\
\text { activities, strength activities, and } \\
\text { flexibility activities. }\end{array}$ & $\begin{array}{l}v \text { high activity } \\
\text { Intermediate: } R R=1.22 \text { (0.91 to } 1.63) \\
\text { Low: } R R=1.73(1.28 \text { to } 2.33)\end{array}$ & $\begin{array}{l}\text { Age, health status, } \\
\text { smoking, and weight }\end{array}$ & \\
\hline $\begin{array}{l}\text { Roger 1998, USA } 25 \\
\text { (Olmsted county, Minnesota) } \\
\text { Retrospective cohort study }\end{array}$ & 741 & $51(15) \dagger$ & $6(2) \dagger$ & 46 & $\begin{array}{l}\text { Physical fitness determined by maximal } \\
\text { exercise test on treadmill, in METs. }\end{array}$ & $\begin{array}{l}\text { For each } 1 \text { MET increase workload } \\
R R=0.75(0.65 \text { to } 0.88)\end{array}$ & Age and comorbidity & $\begin{array}{l}\text { Similar findings were } \\
\text { seen when excluding first } \\
3 \text { years of follow up. }\end{array}$ \\
\hline $\begin{array}{l}\text { Kushi 1997, USA }{ }^{32} \\
\text { Prospective cohort study } \\
\text { (lowa Women's Healthy } \\
\text { Study) }\end{array}$ & 40417 & $55-69$ & 7 & 2260 & $\begin{array}{l}\text { 3-level LTPA index based on combined } \\
\text { frequency and intensity. }\end{array}$ & $\begin{array}{l}v \text { rarely/never participating in moderate activity } \\
1 / \text { week-few/month: } R R=0.71(0.63 \text { to } 0.79) \\
2-4 / \text { week: } R R=0.63(0.56 \text { to } 0.71) \\
>4 / \text { week: } R R=0.59(0.51 \text { to } 0.67) \\
p \text { for trend }<0.001 \\
v \text { rarely/never participating in vigorous activity } \\
1 / \text { week-few/month: } R R=0.83 \text { (0.69 to } 0.99) \\
2-4 / \text { week: } R R=0.74(0.59 \text { to } 0.93) \\
>4 / \text { week: } R R=0.62(0.42 \text { to } 0.90) \\
p \text { for trend }=0.009 \\
v \text { low activity index } \\
\text { Medium: } R R=0.77(0.69 \text { to } 0.86) \\
\text { High: } R R=0.68(0.60 \text { to } 0.77) \\
p \text { for trend }<0.001\end{array}$ & $\begin{array}{l}\text { Age, reproductive factors, } \\
\text { alcohol use, total energy } \\
\text { intake, smoking, estrogen } \\
\text { use, BMl at baseline and } \\
18 \text { years, waist/hip ratio, } \\
\text { high blood pressure, } \\
\text { diabetes, education level, } \\
\text { marital status, and family } \\
\text { history of cancer }\end{array}$ & $\begin{array}{l}\text { Similar findings observed } \\
\text { when excluding women } \\
\text { with CVD or cancer and } \\
\text { first } 3 \text { years of follow-up. } \\
\text { Subjects were post- } \\
\text { menopausal women only. }\end{array}$ \\
\hline $\begin{array}{l}\text { Morgan 1997, UK }{ }^{33} \\
\text { Prospective cohort study } \\
\text { (Nottingham Longitudinal } \\
\text { Study of Activity and } \\
\text { Ageing) }\end{array}$ & 635 & $\geqslant 65$ & 10 & 321 & $\begin{array}{l}\text { Interview, asking about outdoor } \\
\text { productive activities, indoor productive } \\
\text { activities, walking, shopping, leisure } \\
\text { activities, strength activities, and } \\
\text { flexibility activities. }\end{array}$ & $\begin{array}{l}v \text { high activity } \\
\text { Intermediate: } R R=1.53 \text { (1.12 to } 2.09) \\
\text { Low: } R R=2.07 \text { (1.53 to } 2.79)\end{array}$ & $\begin{array}{l}\text { Age, health status, and } \\
\text { smoking }\end{array}$ & \\
\hline $\begin{array}{l}\text { Fraser 1997, USA }{ }^{37} \\
\text { Prospective cohort study } \\
\text { (Adventist Health Study) }\end{array}$ & 1083 & $\geqslant 25$ & $9-11$ & 80 & $\begin{array}{l}2 \text { questions on LTPA (participation in } \\
\text { various vigorous activities } \geqslant 15 \mathrm{~min}, \\
\geqslant 3 \times / \text { week) and OPA ( } 5 \text { options). }\end{array}$ & $\begin{array}{l}v \text { no exercise } \\
\text { Medium: } R R=0.6(0.3 \text { to } 1.8) \\
\text { Vigorous: } R R=0.5(0.3 \text { to } 1.0)\end{array}$ & $\begin{array}{l}\text { Age, adventist, smoking, } \\
\text { diabetes, high blood } \\
\text { pressure, and BMI. }\end{array}$ & $\begin{array}{l}\text { Subjects were black } \\
\text { women only. Exclusion of } \\
\text { subjects with chronic } \\
\text { diseases yielded } \\
\text { non-significant RRs of } \\
1.00,1.08 \text {, and } 0.96 \text {, } \\
\text { respectively, for both } \\
\text { sexes combined. }\end{array}$ \\
\hline $\begin{array}{l}\text { Miilunpalo 1997, } \\
\text { Northeastern Finland }{ }^{16} \\
\text { Prospective cohort study }\end{array}$ & 1500 & $35-63$ & 11 & $\begin{array}{l}244 \\
\text { (both } \\
\text { sexes) }\end{array}$ & $\begin{array}{l}\text { Questionnaire assessing perceived } \\
\text { physical fitness compared with similarly } \\
\text { aged mates; } 3 \text { point scale. }\end{array}$ & $\begin{array}{l}v \text { better } \\
\text { Average: } R R=2.21 \text { (0.85 to } 5.74) \\
\text { Worse: } R R=3.78(1.39 \text { to } 10.28)\end{array}$ & $\begin{array}{l}\text { Age, social status, and } \\
\text { number of physician } \\
\text { contacts }\end{array}$ & \\
\hline $\begin{array}{l}\text { Schroll 1997, Denmark } 34 \\
\text { Prospective cohort study } \\
\text { (Nordic Research on Ageing } \\
\text { Study) }\end{array}$ & 210 & 75 & 5 & 41 & $\begin{array}{l}\text { Assessed by interview and categorised } \\
\text { into sedentary (hardly any physical } \\
\text { activity or mostly sitting with occasional } \\
\text { light activity) or physically active (light } \\
\text { physical exercise around 2-4 } \\
\text { hours/week or more). }\end{array}$ & $\begin{array}{l}v \text { physically active } \\
\text { Sedentary: } R R=1.31 p<0.01\end{array}$ & None & \\
\hline
\end{tabular}




\begin{tabular}{|c|c|c|c|c|c|c|c|c|}
\hline $\begin{array}{l}\text { Author and year, country } \\
\text { study design }\end{array}$ & $\begin{array}{l}\text { No of } \\
\text { women }\end{array}$ & $\begin{array}{l}\text { Age } \\
\text { (years) }\end{array}$ & $\begin{array}{l}\text { Duration } \\
\text { (years)* }\end{array}$ & $\begin{array}{l}\text { No of } \\
\text { deaths }\end{array}$ & Assessment of physical activity & Main resultsł & Factors adjusted for & Other comments \\
\hline $\begin{array}{l}\text { Kampert 1996, USA5 } \\
\text { Prospective cohort study } \\
\text { (Aerobics Center } \\
\text { Longitudinal Study) }\end{array}$ & 6674 & $20-88$ & mean, 8 & 66 & $\begin{array}{l}\text { Physical activity assessed by } \\
\text { questionnaire on walking, running, and } \\
\text { other sports/leisure activities over the } \\
\text { past months. }\end{array}$ & $\begin{array}{l}v \text { no reported activities } \\
1-10 \mathrm{~min} / \text { week: } R R=0.68 \text { (0.39 to } 1.17) \\
11-20 \mathrm{~min} / \text { week: } R R=0.39 \text { (0.09 to } 1.65) \\
\geqslant 21 \mathrm{~min} / \text { week: } R R=1.14(0.27 \text { to } 4.80) \\
p \text { for trend }=0.22\end{array}$ & $\begin{array}{l}\text { Age, examination year, } \\
\text { smoking, chronic illness, } \\
\text { and ECG abnormalities }\end{array}$ & $\begin{array}{l}\text { Fitness data not shown } \\
\text { because identical with } \\
\text { those of Blair et al }\end{array}$ \\
\hline $\begin{array}{l}\text { Kaplan 1996, USA } \\
\text { Prospective cohort study } \\
\text { (Alameda County Study) }\end{array}$ & 3299 & $16-94$ & 28 & 587 & $\begin{array}{l}\text { LTPA index assessed using answers to } \\
\text { three questions on physical exercise, } \\
\text { sports participation, and } \\
\text { walking/swimming. }\end{array}$ & $\begin{aligned} & v \text { lowest LTPA fertile }(T 1) \\
& \text { T2: } R R=0.42 \\
& \text { T3: } R R=0.22\end{aligned}$ & None & $\begin{array}{l}\text { Adjustment for age, sex, } \\
\text { ethnicity, education, } \\
\text { health conditions, and } \\
\text { social isolation still } \\
\text { yeilded significant inverse } \\
\text { associations. }\end{array}$ \\
\hline $\begin{array}{l}\text { LaCroix 1996, USA }{ }^{42} \\
\text { (Seattle, Washington) } \\
\text { Prospective cohort study }\end{array}$ & 1030 & $\geqslant 65$ & 4 & 65 & $\begin{array}{l}\text { LTPA in the previous } 2 \text { weeks assessed } \\
\text { from Modified Minnesota LTPA } \\
\text { questionnaire. }\end{array}$ & $\begin{array}{l}\quad v \text { walked }<1 \text { hour/week } \\
\quad 1-4 \text { hours/week: } R R=0.83 \text { (0.53 to } 1.29) \\
>4 \text { hours/week: } R R=0.91 \text { (0.58 to } 1.42 \text { ) }\end{array}$ & $\begin{array}{l}\text { Age, sex, functional } \\
\text { status, smoking status, } \\
\text { BMI, chronic disease } \\
\text { score, self rated health, } \\
\text { and alcohol consumption }\end{array}$ & $\begin{array}{l}\text { Women were without } \\
\text { severe disability or heart } \\
\text { disease. } \\
\text { Sex-specific results were } \\
\text { not presented for } \\
\text { multivariate analysis. In } \\
\text { crude analysis, p for trend } \\
\text { in women, <0.01. }\end{array}$ \\
\hline $\begin{array}{l}\text { Lissner 1996, Sweden }{ }^{27} \\
\text { (Gothenburg) } \\
\text { Prospective cohort study }\end{array}$ & 1405 & $38-60$ & 20 & 424 & $\begin{array}{l}2 \text { questions on LTPA and OPA in the } \\
\text { previous year, with } 4 \text { response options } \\
\text { for each question, assessed by } \\
\text { questionnaire in 1968-69 and } \\
1974-75 .\end{array}$ & $\begin{array}{l}v \text { low LTPA in } 1968-69 \\
\text { Medium: } R R=0.56 \text { (0.39 to } 0.82) \\
\text { High: } R R=0.45(0.24 \text { to } 0.86) \\
v \text { low } O P A \text { in } 1968-69 \\
\text { Medium: } R R=0.28 \text { (0.17 to } 0.46) \\
\text { High: } R R=0.24(0.14 \text { to } 0.43) \\
\text { Compared with no change in LTPA between 1968-1969 } \\
\text { and 1974-1975 } \\
\text { Increased: RR }=1.11(0.67 \text { to } 1.86) \\
\text { Decreased: } R R=2.07 \text { (1.39 to } 3.09)\end{array}$ & Age & $\begin{array}{l}\text { Women were free of } \\
\text { CVD, cancer, and } \\
\text { diabetes. } \\
\text { Findings little changed } \\
\text { with additional } \\
\text { adjustment for smoking, } \\
\text { alcohol use, education, } \\
\text { BMl, waist/hip ratio, diet, } \\
\text { blood pressure, blood } \\
\text { lipids, and peak } \\
\text { expiratory flow. }\end{array}$ \\
\hline $\begin{array}{l}\text { Mensink 1996, Germany } 18 \\
\text { Prospective cohort study } \\
\text { (German Cardiovascular } \\
\text { Prevention Study) }\end{array}$ & 1212 & $40-69$ & $\begin{array}{l}\text { mean, } 6 \\
\text { range, } 5-8\end{array}$ & 66 & $\begin{array}{l}\text { Frequency and time spent on } 18 \text { leisure } \\
\text { activities during the previous } 3 \text { months. } \\
\text { Each activity assigned a MET score and } \\
\text { classified as total activity, LTPA, } \\
\text { conditioning activity (excluding } \\
\text { walking, cycling, gardening) and sports } \\
\text { activity. }\end{array}$ & $\begin{array}{l}\text { v low total activity } \\
\text { Moderate: } R R=1.24(0.60 \text { to } 2.58) \\
\text { High: } R R=1.29(0.58 \text { to } 2.85) \\
\text { v low LTPA } \\
\text { Moderate: } R R=0.94(0.51 \text { to } 1.75) \\
\text { High: } R R=0.81(0.44 \text { to } 1.49) \\
\text { v low conditioning activity } \\
\text { Moderate: } R R=0.38(0.13 \text { to } 1.06) \\
\text { High: } R R=0.80(0.42 \text { to } 1.54) \\
\text { v no sports activity } \\
\text { < } 1 \text { hour/week: } R R=0.38(0.12 \text { to } 1.23) \\
1-2 \text { hours/week: } R R=0.52(0.23 \text { to } 1.17) \\
>2 \text { hours/week: } R R=0.28(0.07 \text { to } 1.17)\end{array}$ & $\begin{array}{l}\text { Age, systolic blood } \\
\text { pressure, total serum } \\
\text { cholesterol, smoking, and } \\
\text { BMl }\end{array}$ & \\
\hline
\end{tabular}




\section{Table 1 continued}

\begin{tabular}{|c|c|c|c|c|c|c|c|c|c|}
\hline $\begin{array}{l}\text { Author and year, country } \\
\text { study design }\end{array}$ & $\begin{array}{l}\text { No of } \\
\text { women }\end{array}$ & $\begin{array}{l}\text { Age } \\
\text { (years) }\end{array}$ & $\begin{array}{l}\text { Duration } \\
\text { (years) }\end{array}$ & $\begin{array}{l}\text { No of } \\
\text { deaths }\end{array}$ & Assessment of physical activity & Main resultsł & & Factors adjusted for & Other comments \\
\hline $\begin{array}{l}\text { Blair 1996, USA } \\
\text { Prospective cohort study } \\
\text { (Aerobics Center } \\
\text { Longitudinal Study) }\end{array}$ & 7080 & $20-88$ & $\begin{array}{l}\text { mean, } 8 \\
\text { range, } \\
0.1-19\end{array}$ & 89 & $\begin{array}{l}\text { Physical fitness determined by maximal } \\
\text { exercise test on treadmill. Subjects } \\
\text { classified as low (least fit } 20 \% \text { ), } \\
\text { moderate (next } 40 \% \text { ) and high (most fit } \\
40 \% \text { ) fit. }\end{array}$ & $\begin{array}{l}v \text { high fit } \\
\text { Low fit: } R R=2.10 \text { (1.36 to } 3.26)\end{array}$ & & $\begin{array}{l}\text { Age, examination year, } \\
\text { smoking, systolic blood } \\
\text { pressure, cholesterol, } \\
\text { parental history of CHD, } \\
\text { BMl, fasting glucose, } \\
\text { abnormal ECG, and } \\
\text { chronic illness }\end{array}$ & $\begin{array}{l}\text { Most women were } \\
\text { apparently healthy at } \\
\text { baseline. } 350 \text { had } \\
\text { abnormal ECG, and } 958 \\
\text { reported a history of } \\
\text { chronic illnesses (MI, } \\
\text { stroke, hypertension, DM, } \\
\text { or cancer). }\end{array}$ \\
\hline $\begin{array}{l}\text { Ruigómez 1995, Spain }{ }^{39} \\
\text { Prospective cohort study } \\
\text { (Health Interview Survey of } \\
\text { Barcelona) }\end{array}$ & 749 & $\geqslant 65$ & 5 & 109 & $\begin{array}{l}\text { Face to face home interviews. Total } \\
\text { physical activity estimated from single } \\
\text { question and categorised as active } \\
\text { ("doing a job that requires a great deal } \\
\text { of physical effort" or "walking } \\
\text { frequently during the day") or } \\
\text { sedentary ("expending most part of } \\
\text { their day-time standing up but not } \\
\text { walking" or "sitting most of the day"). }\end{array}$ & $\begin{array}{l}\text { vactive } \\
\text { Sedentary: } R R=1.30 \text { (0.77 to } 2.18 \text { ) }\end{array}$ & & $\begin{array}{l}\text { Age, sex, education, and } \\
\text { perceived health status }\end{array}$ & \\
\hline $\begin{array}{l}\text { Sherman 1994, USA }{ }^{11} \\
\text { Prospective cohort study } \\
\text { (Framingham Heart Study) }\end{array}$ & 1404 & $50-74$ & 16 & 319 & $\begin{array}{l}\text { Time spent sleeping, resting, or } \\
\text { engaging in light, moderate, or heavy } \\
\text { physical activity assessed by interview. } \\
\text { Composite score (physical activity } \\
\text { index: PAl) computed as sum of hours } \\
\text { per activity level } \times \text { weight factor based } \\
\text { on oxygen consumption. }\end{array}$ & $\begin{array}{l}v \text { lowest activity quartile (Q1) } \\
Q 2: R R=0.93(0.70 \text { to } 1.23) \\
Q 3: R R=0.65(0.47 \text { to } 0.90) \\
Q 4: R R=0.68 \text { (0.49 to } 0.94)\end{array}$ & & $\begin{array}{l}\text { Age, systolic blood } \\
\text { pressure, serum } \\
\text { cholesterol, smoking, } \\
\text { weight, presence or } \\
\text { absence of glucose } \\
\text { tolerance, LVH, COPD, } \\
\text { and cancer }\end{array}$ & $\begin{array}{l}\text { Subjects were free of } \\
\text { CVD at the baseline. } \\
\text { Excluding first } 6 \text { years of } \\
\text { follow up yielded similar } \\
\text { results. }\end{array}$ \\
\hline $\begin{array}{l}\text { Sherman 1994, USA }{ }^{10} \\
\text { Prospective cohort study } \\
\text { (Framingham Heart Study) }\end{array}$ & 189 & $\geqslant 75$ & 10 & 126 & $\begin{array}{l}\text { Time spent sleeping, resting, or } \\
\text { engaging in light, moderate, or heavy } \\
\text { physical activity assessed by interview. } \\
\text { Composite score (physical activity } \\
\text { index: PAl) computed as sum of hours } \\
\text { per activity level } \times \text { weight factor based } \\
\text { on oxygen consumption. }\end{array}$ & $\begin{array}{l}v \text { lowest activity quartile (Q1) } \\
Q 2: R R=0.70(0.38 \text { to } 1.29) \\
Q 3: R R=0.26(0.12 \text { to } 0.55) \\
Q 4: R R=0.39(0.20 \text { to } 0.77)\end{array}$ & & $\begin{array}{l}\text { Age, systolic blood } \\
\text { pressure, serum } \\
\text { cholesterol, smoking, } \\
\text { weight, presence or } \\
\text { absence of glucose } \\
\text { tolerance, LVH, COPD, } \\
\text { and cancer }\end{array}$ & $\begin{array}{l}\text { Excluding first } 6 \text { years of } \\
\text { follow up yielded similar } \\
\text { results. } \\
\text { Subjects were free of } \\
\text { CVD at the baseline. }\end{array}$ \\
\hline $\begin{array}{l}\text { Davis 1994, USA }{ }^{29} \\
\text { Prospective cohort study } \\
\text { (NHANES I Follow-up Study) }\end{array}$ & 3197 & $45-74$ & 10 & 673 & $\begin{array}{l}2 \text { questions with } 3 \text { response options } \\
\text { each } \\
\text { (1) non-recreational activity: "In your } \\
\text { usual day, aside from recreation are } \\
\text { you physically very active, moderately } \\
\text { active, or quite inactive?" } \\
\text { (2) recreational activity: "Do you get } \\
\text { much, moderate, or little or no exercise } \\
\text { in the things you do for recreation?" }\end{array}$ & 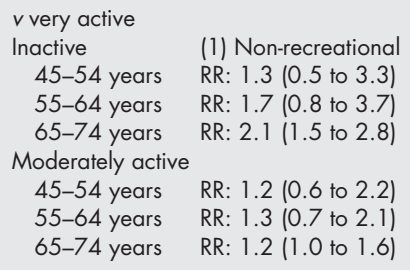 & $\begin{array}{l}\text { (2) Recreational } \\
0.9(0.4 \text { to } 2.4) \\
2.2(0.8 \text { to } 6.6) \\
1.0(0.7 \text { to } 1.4) \\
0.8(0.3 \text { to } 2.3) \\
1.7(0.6 \text { to } 5.2) \\
0.8(0.5 \text { to } 1.1)\end{array}$ & $\begin{array}{l}\text { Smoking, alcohol, BMI, } \\
\text { age, race, education, } \\
\text { income, employment } \\
\text { status, living } \\
\text { arrangement, diet quality, } \\
\text { and chronic conditions }\end{array}$ & \\
\hline $\begin{array}{l}\text { Blair 1993, USA } \\
\text { Prospective cohort study } \\
\text { (Aerobics Center } \\
\text { Longitudinal Study) }\end{array}$ & 3120 & $\begin{array}{l}\text { mean, } \\
40.9\end{array}$ & mean, 8 & 43 & $\begin{array}{l}\text { LTPA assessed by questionnaire asking } \\
\text { about } 18 \text { common activities over the } \\
\text { past months. } \\
\text { Subjects with no activities were } \\
\text { classified as inactive, those who } \\
\text { walked, iogged, or ran as highly } \\
\text { active, and all others as moderately } \\
\text { active. }\end{array}$ & $\begin{array}{l}v \text { high LTPA } \\
\text { Moderate: } R R=0.75 \text { (0.41 to } 1.39) \\
\text { Low: } R R=0.95 \text { (0.54 to } 1.70) \\
p \text { for trend }=0.31\end{array}$ & & Age & $\begin{array}{l}\text { Fitness data not shown } \\
\text { because identical with } \\
\text { those of Blair et al. } .^{8}\end{array}$ \\
\hline
\end{tabular}




\begin{tabular}{|c|c|c|c|c|c|c|c|c|}
\hline $\begin{array}{l}\text { Author and year, country } \\
\text { study design }\end{array}$ & $\begin{array}{l}\text { No of } \\
\text { women }\end{array}$ & $\begin{array}{l}\text { Age } \\
\text { (years) }\end{array}$ & $\begin{array}{l}\text { Duration } \\
\text { (years) })^{*}\end{array}$ & $\begin{array}{l}\text { No of } \\
\text { deaths }\end{array}$ & Assessment of physical activity & Main resultsł & Factors adjusted for & Other comments \\
\hline $\begin{array}{l}\text { Weyerer 1993, Germany }{ }^{19} \\
\text { Prospective cohort study } \\
\text { (Upper Bavarian Field Study) }\end{array}$ & 844 & $\geqslant 5$ & 5 & 41 & $\begin{array}{l}\text { LTPA assessed by interview with single } \\
\text { question, "How often do you currently } \\
\text { exercise for sports (never, occasionally, } \\
\text { or regularly)?" }\end{array}$ & $\begin{array}{l}v \text { regular LTPA } \\
\text { Occasional: RR }=1.14(0.24 \text { to } 5.30) \\
\text { Never: } R R=1.51 \text { (0.41 to } 5.54)\end{array}$ & $\begin{array}{l}\text { Age, social class, } \\
\text { physical and psychiatric } \\
\text { disorders }\end{array}$ & \\
\hline $\begin{array}{l}\text { Rehm 1993, Germany }{ }^{20} \\
\text { Prospective cohort study } \\
\text { (Upper Bavarian Field Study) }\end{array}$ & 778 & $\geqslant 15$ & 13 & 118 & $\begin{array}{l}\text { LTPA assessed by interview with single } \\
\text { question, "How often do you currently } \\
\text { exercise for sports (never, occasionally, } \\
\text { or regularly)?" }\end{array}$ & $\begin{array}{l}v \text { never } \\
\text { Occasional: } R R=0.69(0.33 \text { to } 1.46) \\
\text { Regular: } R R=0.50(0.20 \text { to } 1.25)\end{array}$ & $\begin{array}{l}\text { Age and physicians' } \\
\text { judgment of the severity } \\
\text { of somatic disorders } \\
\text { within the last year }\end{array}$ & $\begin{array}{l}\text { Differences in sample size } \\
\text { from those of Weyerer } \\
\text { et al (1993) } 19 \text { due to } \\
\text { available information on } \\
\text { vital status. }\end{array}$ \\
\hline $\begin{array}{l}\text { C-Claude } 1993, \text { Germany }{ }^{21} \\
\text { Prospective cohort study } \\
\text { (German Vegetarian Study) }\end{array}$ & 1046 & $\geqslant 10$ & 11 & $\begin{array}{l}225 \\
\text { (both } \\
\text { sexes) }\end{array}$ & $\begin{array}{l}\text { Total physical activity classified as low, } \\
\text { medium and high by self evaluation. }\end{array}$ & $\begin{array}{l}v \text { low physical activity } \\
\text { Medium or high: } R R=0.43(0.22 \text { to } 0.82)\end{array}$ & $\begin{array}{l}\text { Age, adherence to } \\
\text { vegetarianism, duration } \\
\text { of vegetarianism, and } \\
\text { BMl }\end{array}$ & $\begin{array}{l}\text { Similar decreases for } \\
\text { CHD, but not cancer } \\
\text { mortality. }\end{array}$ \\
\hline $\begin{array}{l}\text { Rakowski 1992, USA } 35 \\
\text { Prospective cohort study } \\
\text { (US Longitudinal Study of } \\
\text { Aging) }\end{array}$ & 3679 & $\geqslant 70$ & 5 & 555 & $\begin{array}{l}\text { LTPA assessed by responses to } \\
\text { questions: } \\
\text { "How often do you walk a mile or more } \\
\text { at a time, without resting?" and "Do } \\
\text { you follow a regular routine of physical } \\
\text { exercise?" }\end{array}$ & $\begin{array}{l}v \text { walk } \geqslant 4 \text { days } / \text { week } \\
2-3 \text { days } / \text { week: } R R=1.72 \text { (1.01 to } 2.05) \\
1 \text { day } / \text { week: } R R=1.48(0.70 \text { to } 3.10) \\
<1 \text { day/never: } R R=2.49 \text { (1.64 to } 3.80) \\
v \text { have exercise routine } \\
\text { No: } R R=1.32 \text { (1.03 to } 1.69)\end{array}$ & $\begin{array}{l}\text { Age, sex, race, } \\
\text { education, living } \\
\text { arrangement, self rated } \\
\text { health, social } \\
\text { involvement, heart } \\
\text { condition, hypertension, } \\
\text { stroke, diabetes, } \\
\text { functional status, and BMI }\end{array}$ & \\
\hline $\begin{array}{l}\text { Blair 1989, USA } \\
\text { Prospective cohort study } \\
\text { (Aerobics Center } \\
\text { Longitudinal Study) }\end{array}$ & 3120 & $\begin{array}{l}\text { mean, } \\
40.9\end{array}$ & mean, 8 & 43 & $\begin{array}{l}\text { Physical fitness determined by maximal } \\
\text { exercise test on treadmill; women } \\
\text { categorszed into quintiles. }\end{array}$ & $\begin{array}{l}v \text { highest fitness quintile (Q5) } \\
\text { Q4: } R R=1.07(0.55 \text { to } 2.23) \\
\text { Q3: } R R=1.07(0.55 \text { to } 2.09) \\
\text { Q2: } R R=1.45(0.80 \text { to } 2.62) \\
\text { Q1: } R R=1.98(1.13 \text { to } 3.47) \\
\text { p for trend }<0.05\end{array}$ & $\begin{array}{l}\text { Age, serum cholesterol } \\
\text { level, systolic blood } \\
\text { pressure, BMI, smoking, } \\
\text { parental death from CHD, } \\
\text { and serum glucose level }\end{array}$ & $\begin{array}{l}\text { Women were free of } \\
\text { chronic diseases and } \\
\text { abnormal ECG at } \\
\text { baseline. }\end{array}$ \\
\hline $\begin{array}{l}\text { Garfinkel 1988, USA } \\
\text { Prospective cohort study } \\
\text { (Cancer Prevention Study II) }\end{array}$ & 496284 & $\begin{array}{l}\text { mean, } \\
58\end{array}$ & 2 & 2865 & $\begin{array}{l}\text { "How much exercise do you get (work } \\
\text { or play)?" with } 4 \text { response options. }\end{array}$ & $\begin{array}{l}\text { Exercise inversely related to mortality in both } \\
\text { smokers and nonsmokers; exact results not provided. }\end{array}$ & Age, relative weight & \\
\hline $\begin{array}{l}\text { Lapidus 1986, Sweden } \\
\text { (Gothenburg) } \\
\text { Prospective cohort study }\end{array}$ & 1424 & $38-60$ & 12 & 75 & $\begin{array}{l}2 \text { questions on LTPA and OPA in the } \\
\text { previous year, with } 4 \text { response options } \\
\text { for each question, assessed by } \\
\text { questionnaire. }\end{array}$ & $\begin{array}{l}v 3 \text { highest categories of LTPA or OPA } \\
\quad \text { Least active category of LTPA: RR }=1.9(1.1 \text { to } 3.2) \\
\text { Least active category of OPA: RR }=5.2(2.8 \text { to } 9.7)\end{array}$ & Age & Women were free of Ml. \\
\hline $\begin{array}{l}\text { Salonen 1982, Eastern } \\
\text { Finland }{ }^{17} \\
\text { Prospective cohort study }\end{array}$ & 3784 & $35-59$ & 7 & 75 & $\begin{array}{l}2 \text { questions on LTPA and OPA in the } \\
\text { previous year, with } 4 \text { response options } \\
\text { for each question, assessed by } \\
\text { questionnaire. }\end{array}$ & $\begin{array}{l}v 2 \text { highest levels of LTPA or OPA } \\
2 \text { lowest levels of LTPA: RR }=1.6 \text { (1.0 to } 2.3) \\
2 \text { lowest levels of OPA: RR }=2.2 \text { (1.5 to } 3.3)\end{array}$ & $\begin{array}{l}\text { Age, serum cholesterol, } \\
\text { diastolic blood pressure, } \\
\text { BMl, and smoking }\end{array}$ & $\begin{array}{l}\text { Women were free of } \\
\text { AMl, angina, and stroke } \\
\text { in the preceding } 12 \\
\text { months. } \\
90 \% \text { confidence intervals } \\
\text { provided. }\end{array}$ \\
\hline
\end{tabular}

\section{Table 1 continued}




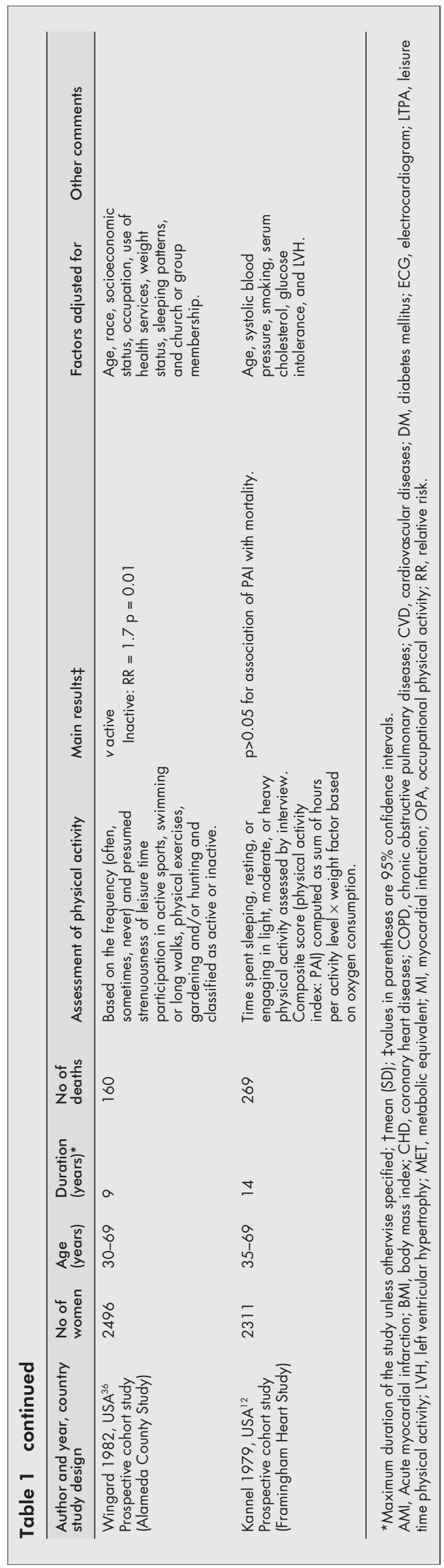

exceeds 34 because some studies had both measures). Only six of the 23 studies that measured leisure time physical activity also measured occupational physical activity ${ }^{176-28}$ or nonrecreational physical activity. ${ }^{23}$ Of the studies examining physical fitness, three used a maximal exercise test on a treadmill. ${ }^{6825}$

\section{Are higher levels of physical activity or fitness} associated with lower death rates in women?

The first question we sought to answer was whether women who are physically active or physically fit postpone mortality compared with their less active or less fit peers. Of the 38 studies included in this review, 23 reported significant inverse associations between physical activity (or physical fitness) and death rate in at least one subgroup. ${ }^{6-11}$ 14-1721 23 25-36 Another nine studies observed at least a $20 \%$ difference in death rates between the most and least active women, which was not statistically significant during the period of study. ${ }^{18-20} 222437-40$ The lack of statistical significance may have been due, in part, to the small number of deaths in these studies. In the remaining six studies, investigators reported less than a $20 \%$ difference in death rates during follow up between the most and least active (or fit) women. ${ }^{5712134142}$

We calculated the median relative risk, comparing the most with the least active (or fit) women, from all 38 studies to estimate an average magnitude of effect of physical activity or fitness on mortality. For all 38 studies, the median relative risk was 0.66 (table 2) - that is, the most active or fit women in these studies were $34 \%$ less likely, on average, to die during the period of study. We then estimated the median relative risk separately for studies of total physical activity, leisure time physical activity, occupational or non-recreational physical activity, and physical fitness. Based on 11 studies, the median relative risk for women who were most active with regard to total physical activity, compared with those who were least

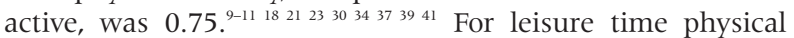
activity (23 studies), the corresponding median relative risk was $0.66 .^{5}{ }^{713-15}$ 17-20 22-24 26-29 31-33 35363842 This figure was 0.54 for occupational or non-recreational physical activity (six studies), ${ }^{17} 2326-29$ and 0.55 for physical fitness (six studies). ${ }^{6} 814162225$ Because the latter two estimates were based on only a small number of studies, there is less confidence in these values.

\section{Is the magnitude of association similar to that seen in men?}

To examine whether the association of physical activity or fitness with all cause mortality in women is of similar magnitude to that seen in men, we conducted a similar exercise for studies conducted in men (reviewed in references 4 , 43,44 ; and references $6,9,13,14,16,17,20,21,29-31,36,37$, $39,45,46)$. The median relative risk for all cause mortality in men, comparing most active with least active, was 0.65 based on all studies of men that we identified $(n=67)$. For studies of total physical activity, this was 0.66 ( 17 studies), for leisure time physical activity, it was 0.70 (30 studies), for occupational or non-recreational physical activity, it was 0.65 ( 10 studies), and for physical fitness, it was 0.55 (10 studies). These data indicate similar associations in both sexes.

\section{Is there a dose-response relation?}

To assess whether a dose-response relation exists, we examined papers in which investigators categorised subjects into at least three levels of physical activity or fitness. We identified 28 such studies. Sixteen of these showed evidence (not always statistically evaluated by the authors) of an inverse dose-response relation between physical activity (or fitness) and all cause mortality in at least one subgroup of

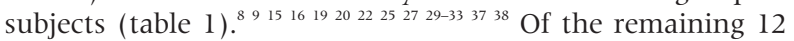
studies, eight reported data compatible with a $\mathrm{J}$ shaped or 
Table 2 Relative risks* (RR) of all cause mortality in women according to physical activity or physical fitness

\begin{tabular}{|c|c|c|}
\hline & No of studies† & Median RR* \\
\hline All studies & 38 & 0.66 \\
\hline Studies of physical activity & 34 & 0.68 \\
\hline Total physical activity & 13 & 0.75 \\
\hline Leisure time physical activity & 23 & 0.66 \\
\hline Occupational or non-recreational physical activity & 6 & 0.54 \\
\hline Studies of physical fitness & 6 & 0.55 \\
\hline
\end{tabular}

reverse J shaped curve, ${ }^{5101114232642}$ and four observed non-significant associations of physical activity with all cause mortality. ${ }^{18} 243541$

For a more formal examination of the dose-response relation, we assessed studies that performed statistical tests for a trend of declining all cause death rates with increasing volume of physical activity (or fitness). Of 13 studies identified, eight observed significant $p$ values for trend..$^{8-11} 15232532$ The remaining five studies reported non-significant trends. ${ }^{5} 7244142$

\section{How much physical activity is required?}

To address this question, we first investigated the volume (amount) of energy expenditure associated with significantly lower death rates in women. Because physical activity was assessed very differently in the various studies, it was difficult to compare volume of energy expenditure across studies. We attempted to make comparisons across studies by estimating the volume of energy expenditure as $\mathrm{kJ}(\mathrm{l} \mathrm{kcal} \approx 4.2 \mathrm{~kJ})$ or MET hours ( 1 MET is the energy expended while resting quietly; it is equivalent to $4.2 \mathrm{~kJ} / \mathrm{kg}$ body weight per hour) in studies in which sufficient data were provided.

Significantly lower death rates were observed at these approximate volumes of energy expenditure: 1050-6720 $\mathrm{kJ} /$ week (250-1600 kcal/week) or 0.5-3.5 MET hours/day ${ }^{32}$; $2100 \mathrm{~kJ} /$ week (500 kcal/week) or 1 MET hour/day ${ }^{27}$; $2100-2940 \mathrm{~kJ} /$ week $(500-700 \mathrm{kcal} /$ week) or 1.0-1.5 MET hours/day ${ }^{26} ; 4200 \mathrm{~kJ} /$ week (1000 kcal/week) or $2.2 \mathrm{MET}$ hours/day ${ }^{15} ; 5460 \mathrm{~kJ} /$ week $(1300 \mathrm{kcal} /$ week $)$ or $2.8 \mathrm{MET}$ hours/day ${ }^{23} ; 5880 \mathrm{~kJ} /$ week (1400 kcal/week) or $3 \mathrm{MET}$ hours/day ${ }^{11} ; 9660 \mathrm{~kJ} /$ week (2300 kcal/week) or 5 MET hours/ day. ${ }^{10}$

We next examined what intensity of physical activity is required for lower death rates. Few studies collected or analysed data on intensity of physical activity, precluding firm conclusions. In the Iowa Women's Health Study, ${ }^{32}$ participation in moderate intensity physical activity was associated with lower death rates. However, in the Canada Fitness Survey, ${ }^{22}$ non-vigorous physical activity was not associated with lower death rates, but an inverse association, of borderline significance, was observed with vigorous physical activity. In the Copenhagen City Heart Study, ${ }^{26}$ participation in sports was associated with additional risk reduction among women who were already participating in other activities of light, moderate, or vigorous intensity.

Several studies examined frequency ${ }^{2032}{ }^{35}$ or duration $^{518}{ }^{42}$ of physical activity in relation to all cause mortality. However, because these studies did not control for the volume of energy expenditure, the findings on frequency or duration essentially reflect the findings for volume of energy expended. Therefore, the independent associations with frequency or duration could not be evaluated in this review.

\section{Is there effect modification by age?}

Finally, we were interested in whether physical activity was beneficial for all cause mortality in both younger and older women. We identified 11 studies ${ }^{14-1726-29} 3^{41}$ in which data were presented separately for women aged $<65$ years at baseline and 13 studies $1013242629-353942$ in which data were provided for those aged $\geqslant 65$ years at baseline. In women aged $<65$ and $\geqslant 65$ years, the median relative risk comparing most active with least active women was 0.67 and 0.58 , respectively. The use of other age cut off points-for example, 55 or 60 yearsproduced similar relative risks for both younger and older women. In six studies, investigators provided findings separately for women of different ages, ranging from 20 to $\geqslant 75$ years. ${ }^{20} 2629324142$ In all but one study ${ }^{29}$ the magnitude of risk reduction was lower among older women than among younger women.

\section{DISCUSSION}

There is clear evidence from 38 studies conducted primarily in the United States and Europe that physically active women postpone mortality compared with inactive women. Formal meta-analysis using raw data from investigators is beyond the scope of this review; instead we conducted a systematic qualitative review using published data. This review indicates that the median risk reduction over the period of study is $34 \%$, a magnitude of association that closely parallels that for men.

Most studies reviewed reported at least a 20\% difference in death rate between the most active and least active women, and most results were statistically significant. In the six studies that did not find an association, physical assessments may not have been sufficiently precise or valid. Although many other studies also suffered from similar limitations in physical activity measurement, they still reported significant results. Therefore the reported findings are likely to underestimate the magnitude of the true association.

Although 28 studies classified women into at least three levels of physical activity, allowing for assessment of dose-response, only 13 formally tested for a trend of declining all cause mortality with increasing levels of physical activity. Eight observed significant inverse trends. Future studies of physical activity and mortality in women need to use measurements that allow a more detailed analysis of dose-response effects. Whether the apparent inverse association follows a linear or non-linear trend also merits consideration, as few studies have examined this.

Because the prevalence of physical inactivity is high, ${ }^{3}{ }^{47}$ an issue of public health importance is the minimum amount of physical activity required to postpone early mortality in women. Few published studies have provided specific estimates of energy expenditure, again highlighting the need for better quantitative assessments of physical activity in epidemiological research. We converted various measures of physical activity to $\mathrm{kJ} /$ week or $\mathrm{kcal} /$ week, assuming the average body weight of a woman to be $65 \mathrm{~kg}$. On the basis of studies from which volume of energy expenditure could be estimated, an energy expenditure of about $4200 \mathrm{~kJ} /$ week (1000 kcal/ week) seemed to be sufficient to avert premature mortality. 
This level of physical activity is in line with recommendations from various US organisations that had based these targets primarily on data in men. ${ }^{1-3}$ In fact, a few studies suggest that even lower levels of energy expenditure may be associated with significant reduction in all cause mortality in women. ${ }^{926} 2732$

We also attempted to assess the role of physical activity intensity in postponing mortality in women. Studies of physical activity intensity and mortality in women are limited and inconsistent in their findings. For example, the Iowa Women's Study separately examined moderate and vigorous activity, noting an inverse association between moderate intensity physical activity and mortality. ${ }^{32}$ The inverse association with vigorous intensity physical activity was only of borderline significance, presumably because few women undertook vigorous activities. However, Villeneuve et $a l^{22}$ reported from the Canada Fitness Survey that non-vigorous leisure time physical activity in subjects with no vigorous leisure time physical activity was not associated with death rate. Further studies are needed to clarify the role of physical activity intensity in postponing mortality in women. Studies of physical activity intensity and risk of coronary heart disease in women have yielded more consistent findings. For example, the Nurses' Health Study $^{48}$ and Women's Health Study ${ }^{49}$ both indicate that moderate intensity activities are associated with lower risk of coronary heart disease. No papers have examined whether, with the same level of energy expenditure, activity bouts of shorter duration (and greater frequency) or longer duration (and lesser frequency) have different effects on all cause mortality in women. Several intervention studies have shown that total physical activity may be more important than duration or frequency for coronary risk factors, ${ }^{5051}$ and large scale observational studies are necessary to confirm or refute these findings for clinical end points such as premature mortality.

When we examined the association of physical activity with death rates in women of different ages, we observed that higher levels of physical activity reduce death rates in both younger and older women. These benefits across all ages of women extend beyond just a reduction in premature mortality. Ferrucci et al ${ }^{52}$ notes that higher levels of physical activity are also associated with fewer years of disability before death, helping women to live more enjoyable lives.

Several limitations of the studies reviewed need to be considered. The assessment of physical activity was often based on instruments developed for men. For example, care of children and household chores were often not accounted for. In the Canada Fitness Survey, Weller et $a l^{23}{ }^{53}$ observed that, for women, non-leisure time physical activity comprised most $(82 \%)$ of their daily energy expenditure. In this study, investigators did not find an association between physical activity and death rates when examining only leisure time energy expenditure. However, after taking into account non-leisure activities, an inverse relation was present. This underscores the importance of assessing non-leisure activities, such as those mentioned above. The challenge is to develop reliable and valid instruments for measuring such activities.

Another limitation is that residual confounding by other lifestyle and dietary habits may be a plausible explanation for an inverse association between physical activity and mortality. Physically active women are likely to be healthy in other ways such as eating behaviour, body weight, and smoking. However, many studies did adjust for potential confounders, and continued to observe significant inverse associations. As all the studies reviewed are observational epidemiological studies, a causal relation cannot be definitely established. However, randomised clinical trials, which provide more rigorous data for cause and effect inferences, are unlikely to be conducted because of the lack of feasibility in maintaining compliance with physical activity over the long term needed in studies of mortality.

We also had difficulty assessing minimum levels of physical activity required for averting premature mortality. Different

\section{Take home message}

Although earlier studies have been conducted primarily in men, studies over the last 10 or 20 years provide convincing evidence that physical activity can also avert premature mortality in women. Following current recommendations of at least 30 minutes of moderate intensity physical activity on most days of the week can postpone mortality in women, as well as men.

cut off points for physical activity were used, not all of which allowed interpretation of the amount of physical activity required.

We were interested in the association of physical activity and death rates in women before and after the menopause, but no studies provided this information. When we examined older women separately using the cut off points of 65,60 , or 55 years, as proxies for identifying postmenopausal women, we found that postmenopausal women also showed inverse associations between physical activity and death rates.

Finally, changes in physical activity were typically not considered in the studies reviewed. Lissner et $a l^{27}$ reported that those who decreased their physical activity level were at increased mortality risk relative to those who maintained activity levels. Recent results from the Nurses' Health Study (not included in this review because the study was published outside the time window for inclusion) also support these findings. ${ }^{54}$

Physical inactivity is pervasive: more than $60 \%$ of American women do not engage in the recommended amount of physical activity, and $25 \%$ are not active at all. ${ }^{3}$ Similar levels of inactivity are observed in England: according to the Health Survey for England in $1994,{ }^{47} 60 \%$ of women were underactive and $20 \%$ were inactive. This is unfortunate, as the present review adds to the list of benefits associated with physical activity in postponing premature mortality in women. This review also supports current physical activity guidelines of at least 30 minutes of physical activity of moderate intensity on most, preferably all, days of the week. ${ }^{1-3} 55$ Although these guidelines were developed primarily from data in men, this review supports its applicability for women as well.

\section{ACKNOWLEDGEMENTS}

This work was supported in part by a grant from the Japan Menopausal Society to Y O, a grant from the Robert Wood Johnson Foundation, and grant HL 67429 from the National Heart, Lung, and Blood Institute of the US National Institutes of Health.

\section{Authors' affiliations}

Y Oguma, H D Sesso, I-M Lee, Department of Epidemiology, Harvard School of Public Health, 677 Huntington Avenue, Boston, MA 02115, USA

R S Paffenbarger Jr, Division of Epidemiology, Stanford University School of Medicine, Stanford, CA, USA

\section{REFERENCES}

1 Physical activity and cardiovascular health. NIH Consensus Development Panel on Physical Activity and Cardiovascular Health. JAMA 1996;276:241-6.

2 Pate RR, Pratt M, Blair SN, et al. Physical activity and public health. A recommendation from the Centers for Disease Control and Prevention and the American College of Sports Medicine. JAMA 1995;273:402-7.

3 US Department of Health and Human Services. Physical activity and health: a report of the Surgeon General. Atlanta, GA: US Department of Health and Human Services, Centers for Disease Control and Preventions, and National Center for Chronic Disease Prevention and Health Promotion, 1996.

4 Lee IM, Paffenbarger RS, Hennekens $\mathrm{CH}$. Physical activity, physical fitness and longevity. Aging (Milano) 1997;9:2-11.

5 Kampert JB, Blair SN, Barlow CE, et al. Physical activity, physica fitness, and all-cause and cancer mortality: a prospective study of men and women. Ann Epidemiol 1996;6:452-7. 
6 Blair SN, Kampert JB, Kohl HW, 3rd, et al. Influences of cardiorespiratory fitness and other precursors on cardiovascular disease and all-cause mortality in men and women. JAMA 1996;276:205-10.

7 Blair SN, Kohl HW, Barlow CE. Physical activity, physical fitness, and all-cause mortality in women: do women need to be active? J Am Coll Nutr 1993;12:368-71.

8 Blair SN, Kohl HW, 3rd, Paffenbarger RS, Jr, et al. Physical fitness and all-cause mortality. A prospective study of healthy men and women. JAMA 1989;262:2395-401.

9 Sherman SE, D'Agostino RB, Silbershatz H, et al. Comparison of past versus recent physical activity in the prevention of premature death and coronary artery disease. Am Heart J 1999;138:900-7.

10 Sherman SE, D'Agostino RB, Cobb JL, et al. Does exercise reduce mortality rates in the elderly? Experience from the Framingham Heart Study. Am Heart J 1994; 128:965-72.

11 Sherman SE, D'Agostino RB, Cobb JL, et al. Physical activity and mortality in women in the Framingham Heart Study. Am Heart $J$ 1994; 128:879-84

12 Kannel WB, Sorlie P. Some health benefits of physical activity. The Framingham Study. Arch Intern Med 1979:139:857-61.

13 Hirvensalo M, Rantanen T, Heikkinen E. Mobility difficulties and physical activity as predictors of mortality and loss of independence in the community-living older population. J Am Geriatr Soc 2000;48:493-8

14 Haapanen-Niemi N, Miilunpalo S, Pasanen M, et al. Body mass index, physical activity and low level of physical fitness as determinants of all-cause and cardiovascular disease mortality-16 y follow-up of middle-aged and elderly men and women. Int J Obes Relat Metab Disord 2000;24: 1465-74.

15 Kujala UM, Kaprio J, Sarna S, et al. Relationship of leisure-time physical activity and mortality: the Finnish twin cohort. JAMA 1998;279:440-4.

16 Miilunpalo S, Vuori I, Oja P, et al. Self-rated health status as a health measure: the predictive value of self-reported health status on the use of physician services and on mortality in the working-age population. J Clin Epidemiol 1997:50:517-28.

17 Salonen JT, Puska P, Tuomilehto J. Physical activity and risk of myocardial infarction, cerebral stroke and death: a longitudinal study in Eastern Finland. Am J Epidemiol 1982;1 15:526-37.

18 Mensink GBM, Deketh M, Mul MDM, et al. Physical activity and its association with cardiovascular risk factors and mortality. Epidemiology 1996:7:391-7.

19 Weyerer S. Effects of physical inactivity on all-cause mortality risk in Upper Bavaria. Percept Mot Skills 1993;77:499-505.

20 Rehm J, Fichter MM, Elton M. Effects on mortality of alcohol consumption, smoking, physical activity, and close personal relationships. Addiction 1993;88: 101-12

21 Chang-Claude J, Frentzel-Beyme R. Dietary and lifestyle determinants of mortality among German vegetarians. Int J Epidemiol 1993;22:228-36.

22 Villeneuve PJ, Morrison HI, Craig CL, et al. Physical activity, physical fitness, and risk of dying. Epidemiology 1998;9:626-31

23 Weller I, Corey P. The impact of excluding non-leisure energy expenditure on the relation between physical activity and mortality in women. Epidemiology 1998;9:632-5.

24 Stessman J, Maaravi Y, Hammerman-Rozenberg R, et al. The effects of physical activity on mortality in the Jerusalem 70-Year-Olds Longitudinal Study. J Am Geriatr Soc 2000;48:499-504

25 Roger VL, Jacobsen SJ, Pellikka PA, et al. Prognostic value of treadmill exercise testing: a population-based study in Olmsted County, Minnesota. Circulation 1998;98:2836-41.

26 Andersen LB, Schnohr P, Schroll M, et al. All-cause mortality associated with physical activity during leisure time, work, sports, and cycling to work. Arch Intern Med 2000;160:1621-8.

27 Lissner L, Bengtsson C, Bjorkelund C, et al. Physical activity levels and changes in relation to longevity. A prospective study of Swedish women. Am J Epidemiol 1996:143:54-62.

28 Lapidus L, Bengtsson C. Socioeconomic factors and physical activity in relation to cardiovascular disease and death. A 12 year follow up of participants in a population study of women in Gothenburg, Sweden. $\mathrm{Br}$ Heart J 1986;55:295-301.

29 Davis MA, Neuhaus JM, Moritz DJ, et al. Health behaviors and survival among middle-aged and older men and women in the NHANES I Epidemiologic Follow-up Study. Prev Med 1994;23:369-76.
30 Lee $\mathrm{Y}$. The predictive value of self assessed general, physical, and mental health on functional decline and mortality in older adults. $J$ Epidemiol Community Health 2000;54:123-9.

31 Bath PA, Morgan K. Customary physical activity and physical health outcomes in later life. Age Ageing 1998;27(suppl 3):29-34.

32 Kushi LH, Fee RM, Folsom AR, et al. Physical activity and mortality in postmenopausal women. JAMA 1997;277:1287-92

33 Morgan K, Clarke D. Customary physical activity and survival in later life: a study in Nottingham, UK. J Epidemiol Community Health 1997;51:490-3.

34 Schroll M, Avlund K, Davidsen M. Predictors of five-year functional ability in a longitudinal survey of men and women aged 75 to 80 . The 1914-population in Glostrup, Denmark. Aging (Milano) 1997:9:143-52.

35 Rakowski W, Mor V. The association of physical activity with mortality among older adults in the Longitudinal Study of Aging (1984-1988). J Gerontol 1992;47:M122-9.

36 Wingard DL. The sex differential in mortality rates: demographic and behavioral factors. Am J Epidemiol 1982;115:205-16.

37 Fraser GE, Sumbureru D, Pribis $P$, et al. Association among health habits, risk factors, and all-cause mortality in a black California population. Epidemiology 1997:8:168-74.

38 Kaplan GA, Strawbridge WJ, Cohen RD, et al. Natural history of leisure-time physical activity and its correlates: associations with mortality from all causes and cardiovascular disease over 28 years [see comments]. Am J Epidemiol 1996; 144:793-7.

39 Ruigómez A, Alonso J, Anto JM. Relationship of health behaviours to five-year mortality in an elderly cohort. Age Ageing 1995;24:113-9.

40 Garfinkel L, Stellman SD. Mortality by relative weight and exercise. Cancer 1988;62:1844-50.

41 Dorn JP, Cerny FJ, Epstein LH, et al. Work and leisure time physical activity and mortality in men and women from a general population sample. Ann Epidemiol 1999;9:366-73.

42 LaCroix AZ, Leveille SG, Hecht JA, et al. Does walking decrease the risk of cardiovascular disease hospitalizations and death in older adults? J Am Geriatr Soc 1996:44:113-20.

43 Lee I-M, Skerrett PJ. Physical activity and all-cause mortality: what is the dose-response relation? Med Sci Sports Exerc 200 1;33:S459-71.

44 Paffenbarger RS, Lee IM. Physical activity and fitness for health and longevity. Res Q Exerc Sport 1996;67:S1 1-28.

45 Kristal-Boneh E, Harari G, Melamed S, et al. Association of physical activity at work with mortality in Israeli industrial employees: the CORDIS study. J Occup Environ Med 2000;42:127-35.

46 Smith GD, Shipley M, Batty GD, et al. Physical activity and cause-specific mortality in the Whitehall study. Public Health 2000; 1 14:308-15.

47 Gray R. 6. Physical activity. In: Colhoun H, Prescott-Clarke P, eds. Health Survey for England 1994. A survey carried out on behalf of the department of health. London: HMSO, 1996:175-203.

48 Manson JE, Hu FB, Rich-Edwards JW, et al. A prospective study of walking as compared with vigorous exercise in the prevention of coronary heart disease in women. N Engl J Med 1999;341:650-8

49 Lee I-M, Rexrode KM, Cook NR, et al. Physical activity and coronary heart disease in women. Is "no pain, no gain" passe? JAMA 2001;285: 1447-54.

50 DeBusk RF, Stenestrand U, Sheehan M, et al. Training effects of long versus short bouts of exercise in healthy subjects. Am J Cardiol 1990;65:1010-3.

51 Murphy MH, Hardman AE. Training effects of short and long bouts of brisk walking in sedentary women. Med Sci Sports Exerc 1998;30:152-7

52 Ferrucci L, Izmirlian G, Leveille S, et al. Smoking, physical activity, and active life expectancy. Am J Epidemiol 1999;149:645-53.

53 Weller IM, Corey PN. A study of the reliability of the Canada Fitness Survey questionnaire. Med Sci Sports Exerc 1998;30: 1530-6.

54 Rockhill B, Willett WC, Manson JE, et al. Physical activity and mortality: a prospective study among women. Am J Public Health 2001;91:578-83

55 Killoran A, Fentem P, Casperson C, et al. Moving on: international perspectives on promoting physical activity. London: Health Education Authority, 1994 